years in those without COPD. After adjusting for confounding by sex and stratifying for age, survival was shorter after 1 st MI in patients with COPD; HR 1.37 (1.23 to 1.52, p<0.001) in those with COPD compared to those without COPD (Abstract S96 figure 1). Survival was shorter in those $(n=96)$ who exacerbated within 6 months of their 1st MI; HR 1.72 (1.13 to 2.60, p=0.01).

Conclusions Survival is shorter after an "unanticipated" MI in patients with COPD and patients who exacerbate within 6 months of their MI have an even higher mortality rate.

\section{BMP signalling in pulmonary hypertension S97 BMPR2 R899X KNOCK-IN MICE DEVELOPED AGE-RELATED PULMONARY HYPERTENSION}

doi:10.1136/thoraxjnl-2011-201054b.97

${ }^{1} \mathrm{~L}$ Long, ${ }^{1} \mathrm{X}$ Yang, ${ }^{1} \mathrm{~N}$ W Morrell, ${ }^{2} \mathrm{M}$ Southwood. ${ }^{1}$ University of Cambridge, Addenbrooke's Hospital, Cambridge, UK; ${ }^{2}$ Papworth Hospital, Cambridge, UK

Background Heterozygous germline mutations in the gene encoding the bone morphogenetic protein type II receptor (BMPR2) underlie the majority $(>70 \%)$ of cases of heritable pulmonary arterial hypertension (hPAH) and a variable proportion of idiopathic $\mathrm{PAH}$ $(15 \%-40 \%)$. There are also reports of PAH in patients with mutations in the downstream Smad signalling proteins. However, to date there is no mouse model that mimics the genetic mutations in human disease.

Methods We developed a knock-in mouse harbouring a heterozygous $( \pm)$ human disease causing mutation in BMPR-II: a nonsense mutation in the cytoplasmic tail (R899X) to determine the in vivo physiologic consequences of this $B M P R 2$ mutation. In addition, we crossed this animal with Smad1 \pm knockout mice to determine the effect of additional loss of signalling via this pathway. Haemodynamic, and morphometric data were collected at 3 months and 6 months of age.

Results At 3 months of age pulmonary haemodynamics and vascular morphometry of R899X \pm and Smad1 \pm mice were similar to wild-type littermate controls. In contrast, at 6 months of age R899X \pm and Smad1 \pm mice developed mild pulmonary hypertension with pulmonary vascular remodelling compared with wildtypes. Pulmonary artery smooth muscle cells from R899X \pm mice were hyperproliferative in serum and exhibited defects in Smad signalling in response to BMPs. When R899X \pm mice were crossed with Smad1 \pm animals, double heterozygous mice had significantly

\section{BMPR2 KI R899X x SMAD1 KO}

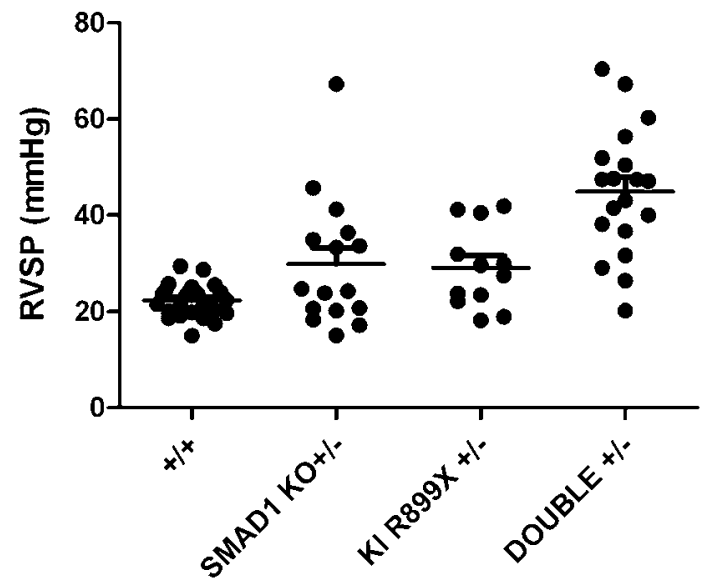

Abstract S97 Figure 1 higher right ventricular systolic pressures than single heterozygous mice.

Conclusion These findings demonstrate that knockin of a human disease causing BMPR-II mutation causes age-related pulmonary hypertension in mice. In addition, we show that the accumulation of defects in the BMP/Smad signalling pathway increases the susceptibility to pulmonary hypertension, highlighting the central role of this pathway in disease.

\section{S98 BMPR-II MUTATIONS DO NOT PREDISPOSE TO PULMONARY ARTERIAL HYPERTENSION IN A MOUSE MODEL OF SCHISTOSOMIASIS}

doi:10.1136/thoraxjnl-2011-201054b.98

A Crosby, E Soon, F Jones, M Southwood, B Dunmore, D Dunne, N W Morrell. Cambridge University, Cambridge, UK

Schistosomiasis is the worldwide leading cause of pulmonary arterial hypertension (PAH) and is particularly prevalent in the thirdworld. More than $80 \%$ of patients with PAH in the western world have a mutation in bone morphogenetic protein type-II receptor (BMPR-II), which is a member of the transforming growth receptor$\beta$ (TGF-b) superfamily and is important in cell proliferation and differentiation. The aim of the study was to determine if mice with a heterozygous null mutation in BMPR-II are more susceptible to schistosomiasis-induced PAH, compared to wild-type littermates.

Methods Wild-type and mutant C56/BL6 mice were infected percutaneously with a low dose of $S$ mansoni. At 17 weeks postinfection right ventricular systolic pressure (RVSP) and right ventricular (RV) hypertrophy, liver and lung egg counts and body weight were measured. Pulmonary vascular remodelling was assessed by morphometry, following immunohistochemistry. Human and mouse pulmonary arterial smooth muscle cells (PASMC) were cultured with $S$ mansoni eggs for $24 \mathrm{~h}$. At $24 \mathrm{~h}$ the expression of cytokines in PASMC were measured by qPCR and cytokine levels in the cell supernatant were measured by ELISA.

Measurements and Main results At 17 weeks post-infection there was no significant difference in RVSP, the degree of RV hypertrophy, liver weight or body weight between wild-type or mutant mice. However, $33 \%$ of the mutant mice died prematurely. After $24 \mathrm{~h}$ coculture with eggs both mouse and human PASMC showed an increase in cytokine expression and cytokine release. More specifically we saw an increase in IL-6, Kc (mouse homologue of IL-8) and IL-13 expression and an increase in IL-6 and Kc secretion. We also saw an increase in PASMC proliferation, determined by Ki67. There was a suggestion that PASMC from mutant mice may display an increase in cytokine response to egg stimulation.

Conclusions This study has shown that BMPR-II mutations do not predispose to schistosomiasis-induced PAH. We have also shown that PASMC respond to $S$ mansoni eggs by an increase in expression and release of inflammatory cytokines. These may play a part in inducing pulmonary vascular remodelling by stimulating PASMC proliferation. However, this affect was not significantly enhanced by BMPR-II mutations.

\section{S99 THE ANTI-MALARIAL DRUG AND LYSOSOMAL INHIBITOR, CHLOROOUINE, INCREASES CELL SURFACE EXPRESSION OF BMPR-II}

doi:10.1136/thoraxjnl-2011-201054b.99

B J Dunmore, L Long, X D Yang, A Crosby, N W Morrell. University of Cambridge, Cambridge, UK

Bone morphogenetic protein receptor type II (BMPR-II) is a member of the transforming growth factor $B$ (TGFß) receptor superfamily. 
Mutations in $B M P R 2$ are the major cause of familial pulmonary arterial hypertension (PAH). Reduced BMPR-II expression is significantly reduced in both familial and idiopathic PAH patients. We have shown previously that BMPR-II expression is regulated via a lysosomal degradative pathway. The anti-malarial drug, chloroquine, blocks lysosomal degradation by raising lysosomal $\mathrm{pH}$ and impairs autophagic protein degradation. Using an experimental rat model of $\mathrm{PAH}$ we observed that chloroquine administration prevented an increase in right ventricular systolic pressure (RVSP), right ventricular hypertrophy (RVH) and vascular remodelling following monocrotaline (MCT) treatment. BMPR-II expression was significantly increased in lungs from chloroquine treated rats. Furthermore, in cellular localisation studies chloroquine increased BMPR-II cell surface expression.

Methods Male Sprague-Dawley rats received a single subcutaneous injection of MCT to induce PAH. To assess prevention or inhibition of $\mathrm{PAH}$ progression, animals received chloroquine, or vehicle, by daily intraperitoneal injection from day 1 to 21 or day 21 to 31 post MCT injections, respectively. Rats were anaesthetised for haemodynamic assessment and lung tissue collected for immunohistochemistry and protein isolation. Muscularisation of small pulmonary arteries was assessed in lung tissue sections by staining with anti-smooth muscle actin. BMPR-II protein expression was determined in frozen lung tissue using western blotting. Cellular localisation of BMPR-II expression in a lung fibroblast cell line that stably expresses green fluorescent protein (GFP) tagged BMPR-II was determined using immunofluorescence and biotinylation of cell surface BMPR-II using NHS-Biotin-SS labelling and precipitation with avidin agarose beads. BMPR-II cell surface expression was then determined by GFP immunoblotting.

Results Chloroquine prevented experimental PAH by significantly decreasing RVSP, RVH and muscularisation in MCT-treated rats. Treatment with chloroquine dramatically increased BMPR-II protein levels in the lung. Furthermore, chloroquine treatment inhibited MCT-induced PAH. This was associated with an increase in cell surface BMPR-II expression in a cell line that stably expresses the GFP-tagged receptor.

Conclusion This study demonstrates the potential use of chloroquine as a therapeutic agent in the treatment of PAH by potentially increasing levels of BMPR-II at the cell surface.

\section{S100 THE BONE MORPHOGENETIC PROTEIN TYPE II RECEPTOR IS CRITICAL FOR VENOUS ANGIOGENESIS IN ZEBRAFISH}

doi:10.1136/thoraxjnl-2011-201054b.100

${ }^{1} \mathrm{~J}$ E Cannon, ${ }^{2} \mathrm{C}$ R Bradshaw, ${ }^{3} \mathrm{~J} \mathrm{C}$ Smith, ${ }^{4} \mathrm{~N}$ W Morrell. ${ }^{1}$ Gurdon Institute and Departments of Medicine and Zoology, University of Cambridge, Cambridge, UK; ${ }^{2}$ Gurdon Institute, University of Cambridge, Cambridge, UK; ${ }^{3}$ MRC National Institute for Medical Research, London, UK; ${ }^{4}$ Department of Medicine, University of Cambridge, Cambridge, UK

Introduction and Objectives Pulmonary arterial hypertension (PAH) is a rare but severe condition, often fatal within 3-5 years due to right heart failure. Mutations in the bone morphogenetic protein (BMP) type II receptor (BMPR-II) underlie heritable forms of the disease but the mechanisms leading to vascular disease remain obscure from studies in mice and humans. Here we use zebrafish, which have a well-documented pattern of angiogenesis, as a model organism to address this question.

Methods The transgenic $\operatorname{Tg}(f l i 1 a: e g f p)$ zebrafish line, where the fli1a promoter drives GFP expression in vascular endothelial and blood cells, was used throughout this work. A variety of methods were used to dissect the role of BMP signalling in vascular development including: (i) BMP receptor inhibitors (dorsomorphin and LDN193189), (ii) antisense morpholino oligonucleotides (morpholinos) and (iii) transgenic zebrafish engineered with heat shock inducible dominant-negative BMP receptors. To identify BMP responsive transcripts from vascular endothelial cells in vivo we developed a system to allow FACS isolation of GFP+ve cells dissociated from $\operatorname{Tg}($ flila:egfp) zebrafish following incubation in LDN193189 or DMSO solvent control. The mRNA transcripts in GFP+ve cells were determined using massively parallel (Illumina) sequencing, mapped to the Zv8 zebrafish genome and differences in transcript abundance between LDN193189 and DMSO treated embryos were determined using cufflinks.

Results Inhibition of BMP signalling with LDN193189 in zebrafish embryos after dorso-ventral patterning has occurred blocked venous but not arterial angiogenesis. This phenotype was reproduced in $B M P R 2$ dominant-negative zebrafish and following knockdown of the zebrafish homologues of BMPR2, bmpr2a and bmpr2b with morpholinos. Illumina sequencing identified the BMP responsive genes in vascular endothelial cells and the gene(s) responsible for the venous angiogenic phenotype are being determined by knockdown of these genes with morpholinos.

Conclusion BMP signalling, via $B M P R 2$, is critical for venous but not arterial angiogenesis in zebrafish. BMPR2 mutations are recognised as causative in patients with heritable $\mathrm{PAH}$, where venous involvement is recognised, but also in pulmonary veno-occlusive disease. Our findings provide novel insights onto the potential role of the pulmonary venous system in pulmonary hypertension and implicate abnormal venous angiogenesis as a novel mechanism underlying this disease.

\section{S101 HETEROZYGOUS LOSS OF BMPR-II PREDISPOSES TO INFLAMMATORY CYTOKINE SECRETION AND PULMONARY VASCULAR SMOOTH MUSCLE PROLIFERATION}

doi:10.1136/thoraxjnl-2011-201054b.101

${ }^{1} \mathrm{E}$ Soon, ${ }^{1} \mathrm{~A}$ Crosby, ${ }^{2} \mathrm{M}$ Southwood, ${ }^{2} \mathrm{~J}$ Pepke-Zaba, ${ }^{1} \mathrm{P}$ Upton, ${ }^{1} \mathrm{~N}$ W Morrell. ${ }^{1}$ University of Cambridge, Cambridge, UK; ${ }^{2}$ Papworth Hospital NHS Trust, Cambridge, UK

Introduction Elevated levels of inflammatory cytokines are associated with idiopathic and heritable pulmonary hypertension (PAH) and predict mortality, but their role in the pathobiology of PAH and the underlying mechanisms remain unclear. We investigated whether loss of bone morphogenetic protein receptor type II (BMPR-II), the underlying cause of heritable $\mathrm{PAH}$, predisposes to increased inflammatory cytokine expression and pulmonary vascular remodelling.

Methods Regulation of cytokine expression by lipopolysaccharide (LPS) was studied in two in vitro models: (1) mouse pulmonary artery smooth muscle cells (PASMCs) heterozygous for a null allele in bmpr2 (BMPR2 \pm ), and (2) human PASMCs with a mutation in BMPR2 (BMPR2mut) and their wild-type counterparts. Cytokine production, regulation and effects on the pulmonary vascular system were examined in an in vivo model using the bmpr2 \pm mouse. Results Both mouse and human in vitro models showed that loss of $B M P R 2$ function leads to increased mRNA expression and secretion of interleukin 6 and IL- 8 both at baseline and after stimulation with LPS. This was associated with loss of expression of antioxidant enzymes such as superoxide dismutase 1 (SOD1) and SOD3, which is demonstrable in both mouse bmpr2 \pm and human BMPR2mut cells. Treatment with the superoxide dismutase-mimetic, Tempol, partially reversed the exaggerated cytokine response to LPS but did not affect the underlying baseline increase. We demonstrated increased phospho-Stat3 signalling and pro-proliferative and pro-survival effects of IL-6 in BMPR2mut PASMCs which is not seen in the wildtype. This was confirmed in the in vivo model, as bmpr2 \pm mice demonstrated increased IL- 6 and IL- 8 expression in sera, lung and liver tissue 3 and $24 \mathrm{~h}$ after exposure to $10 \mu \mathrm{g}$ of LPS. A similar pattern of antioxidant enzyme reduction was also seen in lung tissue from these LPS-treated bmpr2 \pm mice. 